\title{
Electrotactile Feedback in a Virtual Hand Rehabilitation Platform: Evaluation and Implementation
}

\author{
Kairu Li, Student member, IEEE, Peter Boyd, Yu Zhou, Student member, IEEE, Zhaojie Ju, Senior Member, IEEE, \\ and Honghai Liu, Senior Member, IEEE
}

\begin{abstract}
Tactile feedback plays an important role in hand manipulation, especially in the grasping process which is one of the major functions of the hand. However, few commercially available prosthetic hands or hand motor function rehabilitation systems are equipped with tactile feedback. The absence of suitable tactile feedback modules leads to an inferior rehabilitation performance with a large burden on user training and compromised usability. Thus, it is challenging and essential to integrate a proper tactile feedback module with the existing hand rehabilitation systems to achieve a better control performance and accelerate the rehabilitation process. This paper focuses on the implementation and evaluation of the electrotactile feedback enhanced rehabilitation system. A virtual hand rehabilitation platform is proposed comprising an sEMG acquisition module, an electrotactile stimulation module, a virtual environment with sEMG-driven human-like hand and numerical feedbacks of grasping force and fingertip deformation, where a closed-loop control is formed. Three different feedback conditions including visual feedback, electrotactile feedback and no feedback are compared based on the proposed platform. Experiments were conducted on ten able-bodied subjects, and multiple quantitative metrics for the rehabilitation performance evaluation including training burden estimation and success rate of tasks were adopted. Results indicate that the integration of electrotactile feedback is helpful to both reduce the rehabilitation duration and improve the virtual grasping success rate in comparison with the no feedback condition while possessing a better practicality over visual feedback.
\end{abstract}

Note to Practitioners: Abstract-This paper is motivated by the problem of hand grasp control for rehabilitation purposes, but it also applies to other hand motor function rehabilitation process. Existing hand motor function rehabilitation approaches generally lack a proper feedback and rely on the heavy burden during user training and the users experience. This paper suggests incorporating electrotactile feedback to improve the efficiency and efficacy of the rehabilitation process. The electrical stimulation is driven by the myoelectric-sensing-based force estimation and encoded in a manual scheme to fit each individual involved. In our work, a virtual hand rehabilitation platform is implemented to verify the feasibility of electrotactile feedback in reducing the burden of user training and improving the rehabilitation performance, which allows the expanding of the current system into a broader spectrum of motor function rehabilitation applications. Experiments on able-bodied subjects suggest that the electrotactile feedback in the proposed virtual hand rehabilitation platform is feasible, but it has not been tested on the limb-impaired subjects and confined to a manual

K. Li, P. Boyd, Z. Ju and H. Liu* are with the School of Computing, University of Portsmouth, Portsmouth, PO1 3HE, UK (e-mail: Kairu.Li@port.ac.uk; Peter.Boyd@port.ac.uk; Zhaojie.Ju@port.ac.uk; Honghai.Liu@port.ac.uk).

Y. Zhou is with the School of Mechanical Engineering, Shanghai Jiao Tong University, Shanghai, 200240, CHN (e-mail: hnllyu@ @jtu.edu.cn). encoding of electrical stimulation. In future research, the design of a general electrotactile feedback enhanced hand rehabilitation platform with a standardised stimulation parameter optimisation will be addressed and further validated on the subjects with limbimpairments and amputation.

Index Terms-Electrotactile feedback, hand rehabilitation, virtual environment, sEMG.

\section{INTRODUCTION}

$\mathbf{T}$ ACTILE sensation of the human hand is important for the exploration and interaction with an environment involving a large variety of tasks, ranging from basic grasps to complex operations of sophisticated instruments. For transradial amputees, the loss of sensations and motor functions due to the amputation can inevitably deteriorate the quality of life and make an individual feel less capable and more dependent [1]. Even if equipped with hand prostheses, transradial amputees still face to huge obstacles in the process of rehabilitation and some situations where fine-force control is necessary due to the absence of tactile feedback [2], which leads to a high rejection rate from prosthesis users. Thus, it is expected to integrate tactile feedback with hand prostheses to improve the user experience and prosthesis performance. Apart from this, tactile feedback has also been reported to be effective on alleviating phantom pain, reducing muscle fatigue and enhancing a sense of body ownership [3][4], which motivates the research in biomedical engineering [5], and the achievements can be further applied to virtual reality [6][7], robotics [8][9][10], etc.

To close the loop of prosthesis control by tactile feedback is an important academic topic in the area of upper-limb rehabilitation. Studies mainly investigated the impacts of tactile feedback on the self-embodiment and prosthesis performance. On one hand, it was revealed that tactile feedback did help to generate a sense of body ownership and improve the user experience [11][12][13][14], and it was something that amputees wanted in their prostheses [3][15]. On the other hand, the opinion of tactile feedback on improving prosthesis performance is still a matter of controversy. Most studies concluded that the integration of tactile feedback improved the performance of prosthesis manipulation [16][17][18], although there were some studies showing an improvement only with certain conditions or users, or even little difference when compared with the non-feedback condition [19]. In some cases, clinical therapists claimed that amputees with only visual and audio feedback could acquire comparable prosthetic 
grasping performance with the performance of a closed-loop condition if the rehabilitation/training process was adequate enough. However, the time-consuming rehabilitation/training process may cost several weeks, months or even more than one year [20][21] and also cause a great load of the cognitive burden not only during the training stage but also in practical use.

Also, the clinical applications of tactile feedback in rehabilitation are still limited, although some efforts have been devoted to the device development and exploring the impact of tactile feedback [22][23]. Few of the commercial hand prostheses provide tactile feedback, neither the rehabilitation system for their users. Consequently, prosthesis users have to rely on some indirect cues (e.g. vision, motor sound and the prosthesis velocity of closing) to compensate the lack of tactile feedback in the prosthesis manipulation [3][24], which requires continuous visual or auditory attention and increase the cognitive burden. Thus, it is expected to take measures to shorten the rehabilitation duration and reduce the cognitive burden during the following daily use.

Virtual environments, as a promising therapeutic training tool, can provide an immersive and enjoyable treatment display for upper limb rehabilitation [25] and act as a flexible platform that can be customised to meet the individual needs [26]. Especially for circumstances requiring fine-control, such as grasping eggs, people can practice in a virtual environment without creating any waste or causing any damage. Together with tactile feedback, the virtual environment can provide an enriched training environment and enhance the sense of interactivity [27], which may boost the user's involvement and perceptive capability for a better rehabilitation performance. Some efforts have been made to apply tactile feedback to handrelated tasks in virtual environments. An array electrode of 15 stimulation levels was proposed to compare the performance of two coding schemes on the control of the prosthesis grasping force. A simplified gripper was employed in a visual interface, which was not as flexible and interactive as a human-like hand [28]. Additionally, tactile feedback was utilised to enhance virtual reality experience [27] and reduce phantom pain [6], however, the impact of tactile feedback on hand rehabilitation process based on virtual reality has not been investigated.

To investigate the impact of tactile feedback on the performance of grasping control, an sEMG-driven virtual rehabilitation platform integrated with a portable electrotactile stimulator is established. The grasping performance with electrotactile feedback is compared with that in conditions of visual feedback and non-feedback. The main contributions of this study are: 1) establishing a integrated rehabilitation platform for hand function recovery, which includes an sEMG (surface electromyography) processing device, virtual grasping environment and an electrotactile stimulator; 2) confirming that the integration of tactile feedback is helpful to reduce the rehabilitation time and improve the grasping performance by conducting experiment based on the proposed platform to compare the performance with that in conditions of visual feedback and non-feedback. The remaining parts of this paper are organized as follows. Section III presents the rehabilitation platform design from both structural aspect and modular aspect. Experimental methods are presented in Section III. Section IV demonstrates the results, followed by a discussion in Section V.

\section{REHABILITATION PLATFORM ESTABLISHMENT}

To validate the hypothesis of the tactile feedback's effectiveness on hand grasping rehabilitation, a novel integrated platform is established locally to support the experiment of virtual grasping in different feedback conditions.

\section{A. Platform construction}

The schematic diagram of the rehabilitation platform is presented in Fig. 1(a) with a detailed decomposition of the virtual environment in Fig. 1(b). According to Fig. 1(a), after being informed about the weight of the target object, the subject will try to conduct grasp gesture with an empty hand. Then the sEMG signal caused by the muscle contraction is detected and processed by an sEMG acquisition module. The output sEMG intensity is sent to a virtual environment where a virtual hand and object are set for grasping display. The rehabilitation process can be switched among three feedback conditions, none feedback (NF), visual feedback (VF) and electrotactile feedback (EF), where different feedback information will be provided to the subject for grasping force control.

Fig. 1(c) presents an experimental setup. Two pairs of sEMG electrodes are attached on a subject's forearm of the left arm. One channel is to extract sEMG signals for grasping force estimation, and the other one acts as a reference signal for noise filtering. The subject will contract their arm muscles when he/she is told or sees an object with a certain weight and force range of safe holding. Taking the visual feedback condition for example, when the force bar is lower than the safe holding range, the subject needs to contract the arm muscles more by grasping harder, and vice versa for when the bar is higher than the safe holding range. Consequently, the sEMG signals generated by muscle contraction will be detected by the sEMG acquisition module, which outputs the intensity of sEMG signals to the following modules.

In the condition of NF, the subject will be asked to close eyes and complete grasps only based on experience. Regarding the VF condition, the subject is allowed to watch a realtime grasping animation, a force bar and a deformation bar displayed via a virtual interface, so that the subject can adjust the muscle contraction accordingly to try for successful grasps. The principle of EF is similar to $\mathrm{VF}$, but the subject receives force feedback according to the electrical stimulation instead of a visual display. Different levels of electrotactile stimulation will be generated by the electrotactile stimulator and delivered to the subject's right arm via three pairs of electrodes. Thus, either of VF or EF will close the loop of grasping force control for the rehabilitation platform.

The close-loop rehabilitation platform is realised by multiple functional modules developed by us, with the details introduced in the following section. 


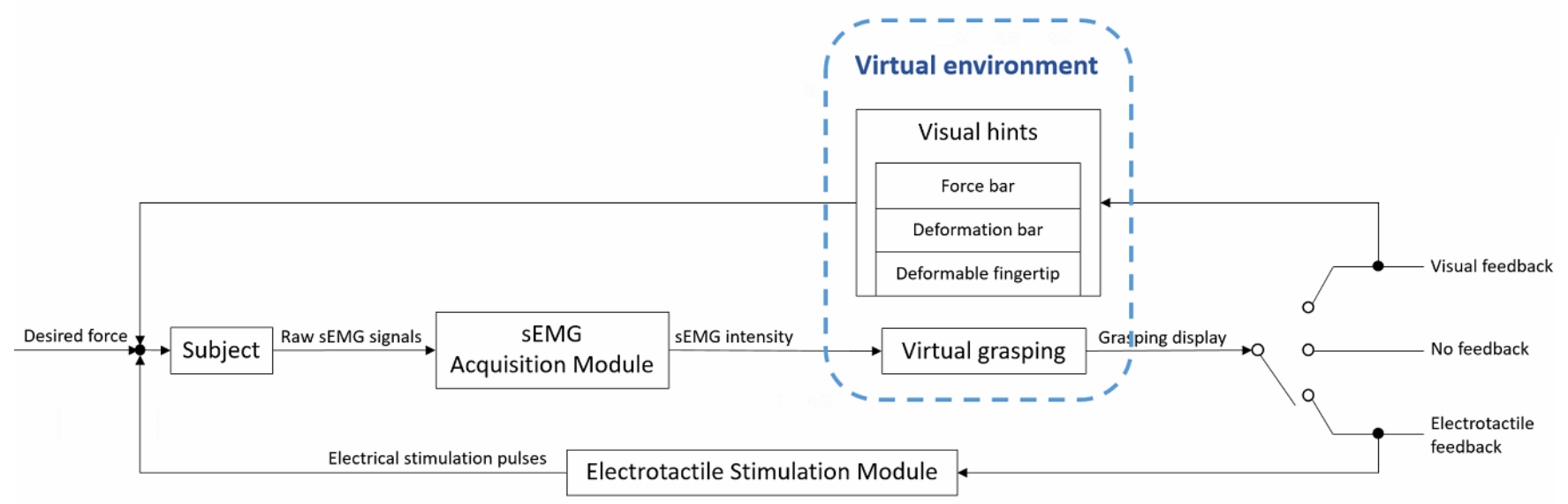

(a) Schematic diagram of the hand rehabilitation platform

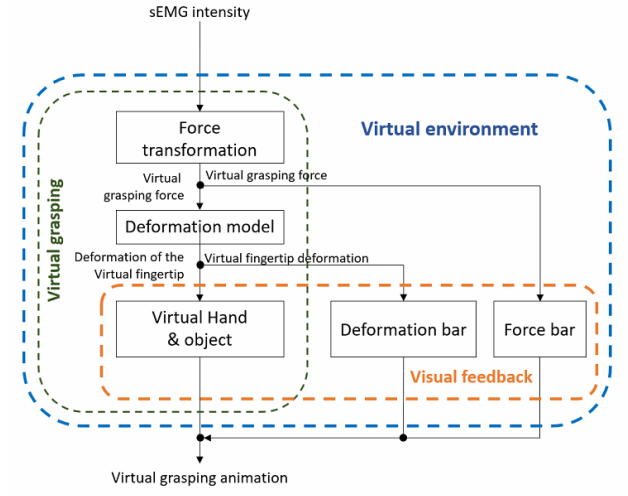

(b) Virtual environment

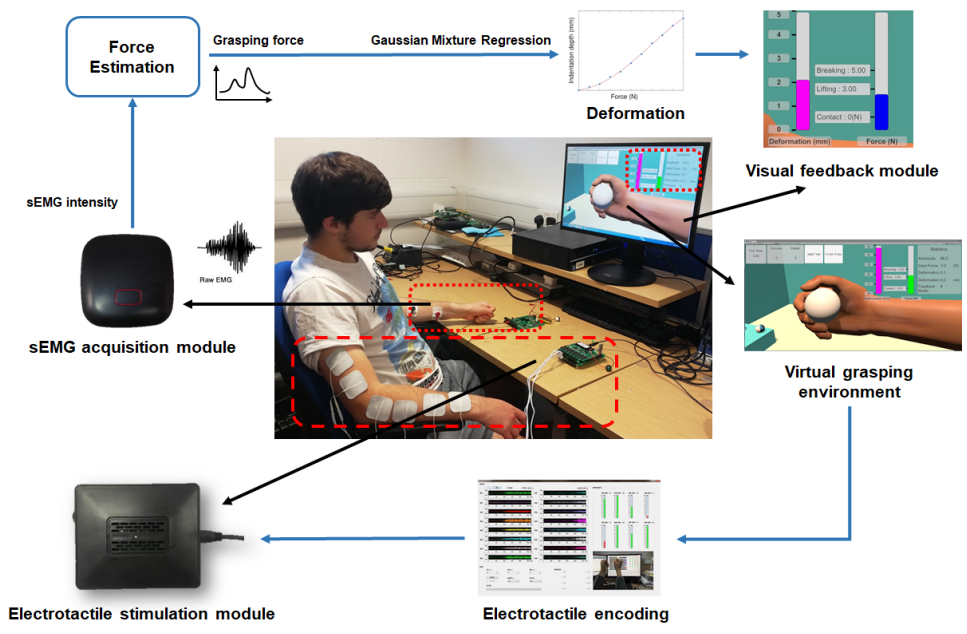

(c) Experimental setup

Fig. 1. Platform construction

\section{B. Functional module description}

The rehabilitation platform consists of an sEMG acquisition module, a virtual hand environment where a fingertip deformation model is implemented and an electrotactile stimulation module.

1) $s E M G$ acquisition module: A multi-channel sEMG acquisition system (Elonxi Ltd, UK) proposed in a previous work is utilised to detect the sEMG signal generated from the subjects arm [29][30]. sEMG signals are the electrical manifestation of the activity of muscle fibers recorded by noninvasive electrodes attached on the human skin. The analysis and interpretation of the sEMG signal can be applied for hand motion recognition, control of smart prosthetic devices, and so on. In this study, sEMG signals are used to control the virtual hand and estimate the virtual grasping force which is proportional to the intensity of sEMG signals. The employed sEMG acquisition system has up to 16 channels for sEMG signal collection which can be applied for gesture classification, neuromuscular disease diagnosis, etc. The sEMG signals are sampled at a frequency of $1 \mathrm{kHz}$. After the integrated signal processing, the sEMG acquisition module package and transmit the value of sEMG intensity to the host computer via a wireless module. In this study, two channels were employed, because only the intensity of sEMG signal was needed and no complex tasks like gesture recognition were involved.

2) Electrotactile stimulation module: Various types of stimulations have been explored to provide tactile feedback in available research, such as electrotactile feedback, vibrotactile feedback and mechanotactile stimulation [31]. Compared with the others, electrotactile feedback has advantages of low power consumption, lightweight, little noise and the potential to recover a natural sense.

An electrotactile stimulator (ETS) proposed in our previous work [32] is employed to provide force feedback in the rehabilitation platform. The stimulator has up to 16-channel outputs with adjustable parameters, including the amplitude $(0-100 \mathrm{~mA})$, frequency $(1-200 \mathrm{~Hz})$ and pulse width $(0-500 \mu \mathrm{s})$. The stimulator can deliver different waveforms of stimulation, while symmetric biphasic square pulses are chosen as the output signal. In biphasic waves, negative pulses can neutralize the charge accumulation on the skin, the polarization effect caused by positive pulses and prevent the tissue damage [33]. In this study, three channels are used to present 9 levels of force intensity. The coding scheme will be detailed in Section III-C. 
3) Virtual hand environment: A visual interface of the rehabilitation platform as shown in Fig. 2 is designed to conduct rehabilitation programme with different feedback conditions by integrating the sEMG module and the ETS module. It also provides visual feedback by displaying a deformable virtual hand, a force bar and a deformation bar. A basic hand simulator was applied in the virtual environment [34]. It was developed into a human-like hand with deformable fingertips during grasping tasks. A ball with adjustable weight and rigidity is set as a grasped object for the practice of fine force control. A force bar shows the grasping force variation and three scales which are the thresholds of contacting, lifting and breaking points, while the deformation bar simultaneously presents the virtual fingertip's deformation depth. The trial results are displayed in the board of upper-left corner, while related statistics are listed in the right.

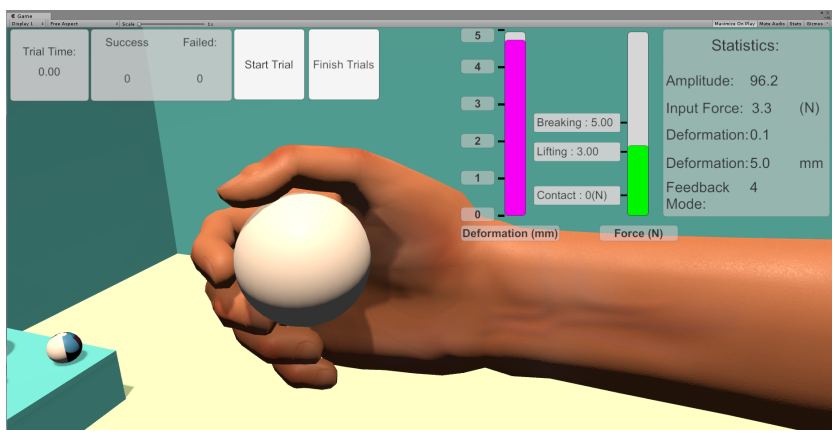

Fig. 2. Virtual grasping interface

The hand pose, grasping force and the fingertip deformation of the virtual hand are controlled based on the intensity of sEMG signals output from the sEMG acquisition module in real time. With the continual increase of the grasping force, the fingertip deformation started to change accordingly within a range of 0-5 $\mathrm{mm}$. To mimic human skin, when the fingertip skin was pressed to a certain level, the fingertip did not deform further, although the contact force kept increasing. A two-hierarchical feedback comprising both grasping force and fingertip deformation is applied. Specifically, the feedback strategy is realised in the form of visual feedback and integrated to our virtual platform.

Grasping force estimation lies on the basis of force prediction in [35], which utilises the EMG signals to fit the exerted force. The model was tuned by the locally captured data of ten subjects. The deformation model of the virtual fingertip is designed according to the human fingertip's biomechanics investigated in our previous work, which proposed an in vivo dataset of the human fingertip and a haptics model to predict the contacting force according to the fingertip deformation depth [36]. The parameters of the haptics model needed to be customised individually. However, in this study, the deformation depth of the virtual fingertip needs to be calculated according to the virtual grasping force which is estimated based on sEMG signals. Additionally, a generalised model with extensive suitability is also expected for the application of the rehabilitation system. Thus, Gaussian mixture regression (GMR) is introduced to calculate the virtual fingertip's deformation.

GMR is a probabilistic regression method which can be applied based on the parameters of a Gaussian mixture model (GMM). GMM is a mixture of a sequence of Gaussian distributions. It is a popular approach to data approximation and allows for a proper trade-off between the variation of the training data and model complexity [37]. A $D$-dimension Gaussian distribution of a vector $\boldsymbol{x}=\left(x_{1}, x_{2}, \ldots, x_{D}\right)^{T}$ is defined by Eq. 1.

$$
p(\boldsymbol{x})=\frac{1}{\sqrt{(2 \pi)^{D}|\Sigma|}} \cdot e^{-\frac{1}{2}\left[(\boldsymbol{x}-\mu)^{\mathrm{T}} \Sigma^{-1}(\boldsymbol{x}-\mu)\right]}
$$

where $\Sigma$ and $\mu$ is the covariance matrix and the mean of the Gaussian distribution.

In this study, a GMM consists of $K$ components of 2 dimension Gaussian distributions defined in Eq. 2 is used to characterise the variations and correlations across the variables of the aforementioned dataset $\xi_{i}=\left\{\xi_{f, i}, \xi_{d, i}\right\}_{i=1}^{N}$. The dataset includes $N$ datapoints, and $K$ is set to 3 given the feature of the dataset. $\xi_{f}$ is the contacting force, $\xi_{d}$ is the deformation depth of the fingertip, $\xi_{f, i}$ and $\xi_{d, i}$ are the corresponding $i$ th values.

$$
p(\xi)=\sum_{k=1}^{K} \omega_{k} \cdot \frac{1}{2 \pi \sqrt{\left|\Sigma_{k}\right|}} \cdot e^{-\frac{1}{2}\left[\left(\xi-\mu_{k}\right)^{\mathrm{T}} \Sigma_{k}{ }^{1}\left(\xi-\mu_{k}\right)\right]}
$$

where $\omega_{k}$ is the prior probability (weight) of the $k$ th Gaussian component, and there is $\sum_{k=1}^{K} \omega_{k}=1$.

To estimate the parameters of the GMM by maximising the log-likelihood, the $k$-means clustering method and the standard expectation-maximization (EM) algorithm are employed for initialization and iteration. Consequently, the mean $\mu$ and the covariance matrix $\Sigma$ of the $k$ th Gaussian component are obtained as Eq. 3.

$$
\mu_{k}=\left\{\begin{array}{ll}
\mu_{f, k} & \mu_{d, k}
\end{array}\right\}, \Sigma_{k}=\left(\begin{array}{cc}
\sigma_{f, k} & \sigma_{f d, k} \\
\sigma_{d f, k} & \sigma_{d, k}
\end{array}\right)
$$

Taking values of $\xi_{f}$ as query data, the corresponding $\xi_{f}$ values are estimated through GMR. The conditional expectation $\widehat{\mu}_{d \mid f, k}$ of $\xi_{d, k}$, given $\xi_{f}$, and the estimated conditional covariance $\widehat{\sigma}_{d, k}$ of $\xi_{d, k}$, given $\xi_{f}$, can be calculated by Eq. 4 .

$$
\begin{aligned}
\widehat{\mu}_{d \mid f, k} & =\mu_{d, k}+\frac{\sigma_{d t, k}\left(\xi_{f}-\mu_{f, k}\right)}{\sigma_{f, k}} \\
\widehat{\sigma}_{d \mid f, k} & =\sigma_{d, k}-\frac{\sigma_{d f, k} \cdot \sigma_{f d, k}}{\sigma_{f, k}}
\end{aligned}
$$

The responsibility $\lambda_{k}$ of the $k$ th Gaussian component for the estimation of $\xi_{f}$ and $\xi_{d}$ is defined as Eq. 5 .

$$
\lambda_{k}=\frac{p\left(\xi_{f} \mid k\right)}{\sum_{k=1}^{K} p\left(\xi_{f} \mid k\right)}
$$

Based on Eq. 4 and Eq. 5, the conditional expectation $\widehat{\mu_{d}}$ of $\xi_{d}$, given $\widehat{\xi_{f}}$, and the conditional covariance $\widehat{\sigma_{d}}$ of $\xi_{d}$, given $\widehat{\xi_{f}}$, can be calculated by Eq. 6 .

$$
\widehat{\mu}_{d}=\sum_{k=1}^{K} \lambda_{k} \widehat{\xi}_{d, k} \quad \widehat{\sigma}_{d}=\sum_{k=1}^{K} \lambda_{k}^{2} \widehat{\xi}_{d, k}
$$


Thus, the deformation of the virtual fingertip $\xi_{d}$ in the condition of the virtual contact force $\xi_{f}$ can be estimated and is equal to $\widehat{\mu}_{d}$ with a constraint of $0-5 \mathrm{~mm}$ as mentioned in Section II-B3.

In summary, the proposed virtual platform integrates biological acquisition module, stimulation module, and the interactive interface, which enables a broader range of applications, such as the biological engineering and virtual reality, although it is only employed for hand rehabilitation in this study.

\section{EXPERIMENTAL SETUP}

The experiment aims to evaluate the impact of electrotactile feedback on the rehabilitation process. Definitions of various virtual grasp results are given firstly in this section. Then, experimental setups of different feedback conditions are presented, followed by a description of the experimental protocol and methods of data analysis.

\section{A. Definition of task success and failure}

The fine control of different force levels was realised by setting different weights of the objects from light, medium to heavy. Each object was set with a certain grasping threshold of lifting and breaking. The light object corresponded to the electrotactile level 2 and level 3 , while the medium object to level 5, level 6 and the heavy object to the electrotactile level 6 , level 7 and level 8. Subjects needed to apply proper grasping forces to hold each object according to its weight.

A successful grasp trial required the subject to maintain the grasping force between the lifting and breaking points (safe holding range) for at least $2 \mathrm{~s}$. Otherwise, it was considered as a failed trial. If the grasping force kept below the lifting threshold for more than $5 \mathrm{~s}$, the subject failed because of a no-lift. If the force went beyond the safe range for more than $300 \mathrm{~ms}$, such as exceeding the breaking threshold or dropping below the lifting threshold, the trial also failed because the object broke or dropped.

\section{B. Visual feedback setup}

The visual feedback was provided by the interface introduced in Section II-B3 and a typical illustration was shown in Fig. 2. At the beginning of each trial, the virtual hand maintained an open pose. It began to close and touch the ball when the virtual grasping force increased from a relaxed state to the contacting threshold. With the increase in the grasping force, the object was lifted, held or broke subsequently. Despite the realised function of visual fingertip deformation in the virtual environment, a more observable hint is desired for the purpose of user training. Thus, the hand-object interaction is also provided numerically by the deformation bar.

Subjects were asked to practice and conduct successful grasps as many as possible. They received visual feedback by observing the force bar and deformation bar which rose or dropped linearly to the virtual grasping force. Subjects could adjust their muscle contracting intensity to control the grasping force. After each trial, the system was set to an initial state with the virtual hand open and subjects relaxed arms.

\section{Electrotactile feedback setup}

Multi-channel Electrotactile feedback was provided by discriminable modes of electrical stimulation in this study to provide feedback of the virtual grasping force. A mixed coding scheme was employed by modulating multiple stimulation parameters, including amplitude, frequency, pulse width and a combination with spacial coding. Two to fifteen outputs were found in existing studies [28]. Considering the cognitive accuracy and perceptive burden, this study employed 9 stimulation output modes to represent the real-time intensity of the sEMG signals/grasping force from light to hard. They were delivered by three pairs of electrodes attached on the subject's right arm. Each pair of electrodes delivered three levels of electrical stimulation from low intensity to high intensity. The stimulation parameters were modulated individually according to the subject's request before the experiment to ensure a comfortable and identifiable perception of each stimulation level. A typical coding scheme applied in the experiment will be provided in Section IV-A. During the experiment, subjects could to control the grasping force according to the stimulation levels by adjust their muscle contracting intensity.

\section{Experimental protocol}

The experiment included three stages, preparation, training and testing. Before the experiment, the experimental aim and procedure were explained to the subjects who then signed the consent form. Ten able-bodied subjects (24-29 years old) participated in the experiment. The study was approved by the local ethical committee.

1) Preparation: The goal of preparation was to get subjects familiar with the rehabilitation system and learn the coding scheme of the electrotactile stimulation. In the beginning, the subject was comfortably seated on an armchair and wore two pairs of sEMG electrodes and three pairs of eletrotactile stimulation electrodes on the left arm and right arm, respectively.

Firstly, the upper limit of sEMG signal intensity was set by asking the subject to contract their left forearm muscles as hard as possible for three times. Sixty percent of the average intensity was regarded as the upper limit of the subject's sEMG signal.

Secondly, the stimulation parameters of 9 electrotactile stimulation levels were determined. An initial setting based on previous experimental experience was tested on the subject's right arm. It would be adjusted according to the subject's verbal feedback. If the subject reported any discomfort or difficulty in distinguishing stimulation levels, the stimulation parameters would be reduced or increased accordingly until the subject could identify all the stimulation levels without discomfort.

Finally, the subject had one minute to experience the rehabilitation system and conduct virtual grasps freely with simultaneously visual feedback and electrotactile feedback.

2) Training and testing: The training and testing process were conducted in three feedback conditions, which included: a) feed-forward control with no feedback (NF); b) closed-loop control with visual feedback (VF); c) closed-loop control with electrotactile feedback (EF). 
- Feed-forward control with no feedback (NF)

During the training process, 3 objects of different weights were provided to a subject for virtual grasping in a sequence of object 1 (light), object 2 (medium) and object 3 (heavy). For each object, the subject had one minute to practical grasping by observing the force bar via the visual interface. Then, the subject was asked to close their eyes and perform the virtual grasping attempts based on their practical experience. After each attempt, the subject was informed about the grasping result, whether the object dropped, broke or successfully held, so that the subject could adjust the arm contracting intensity of the arm in the next attempt. Finally, the subject would learn the fine control of force for the object. After the subject successfully grasped the object twice in a row, another object would be shown to the subject. The total number of attempts for each object in training process was recorded.

In the testing stage, the subject was asked to grasp each object for 10 times. Different from the training process, The objects of different weights were presented in a random order to the subjects during the testing stage. The experiment in the condition of no feedback was completed when the subject accomplished the training and testing on all objects.

- Closed-loop control with visual feedback (VF)

The procedure in this feedback condition was similar to that of the non-feedback condition, but the subject was allowed to watch the visual interface. The subject could perform virtual grasping according to the force bar and observe the grasping results by himself/herself.

- Closed-loop control with electrotactile feedback (EF) Different from the other two conditions, a short preparation needed to be conducted before the training with electrotactile feedback. The stimulation parameters determined in the preparation stage were presented to the subject again. On one hand, it would make sure that the subject could identify each stimulation level and be happy with the settings. On the other hand, it would give the subject a second chance to adjust the parameters if necessary. After the finalisation of the stimulation parameters, the researcher stimulated the subject with different stimulation levels randomly and ask the subject to report the level numbers. The preparation process was accomplished until the subject correctly answered all the stimulation levels in a row. The training and testing experiment with electrotactile feedback was similar with the experiment with no feedback and visual feedback, but the subject was asked to close their eyes and could only tell the grasping force according to the electrotactile stimulation levels.

The whole experiment for each subject lasted for about 2 hours. Subjects might experience muscle fatigue during the training and evaluation process due to the attempts of contracting arm muscles and the electrotactile stimulation. To avoid discomfort and the interference of muscle fatigue, subjects were free to take a rest at any time during the experiment. For both training process and testing process, the attempt result (success/failure), grasping force and time consumption in each attempt for every object were recorded for the evaluation of different feedback conditions.

\section{E. Data analysis criteria}

The rehabilitation performance was evaluated by the following criteria. An initial data processing such as the elimination of the maximum value and the minimum value was conducted before the result analysis.

1) Number of attempts: The number of attempts is the number of grasping trials before the subject successfully grasped an object twice in a row. This also includes the total number of attempts which were required during the training stage. The number of attempts is used to evaluate how fast the subject could learn the fine control of force in different feedback conditions, and how long the training process took.

2) Duration of Training: The duration of training is the sum of each attempt's duration time for each object in the training process. As a complementary value to the number of attempts, the duration of training also aims at the quickness of the training progress.

3) Duration of an attempt: The duration of an attempt is the average time consumed on each attempt during the testing stage. It is used to evaluate the subject's operation speed of the virtual grasp.

4) Success rate: The success rate is the percentage of the successful grasps (without slip or breaking) during the testing stage. The success rate was to evaluate the rehabilitation performance.

\section{RESUlTS}

\section{A. Setting of parameters}

The transient upper limit of the sEMG signal's intensity varied with individuals from $400 \pm 600$. Considering that the subject was required to hold muscle contraction for several seconds, the long-lasting upper limits of subjects were also tested, and they presented little difference with an average of 210.

In contrast, there was a huge individual difference of the settings on electrotactile stimulation parameters because of a different sensitivity to electrical stimulation among subjects. Table I demonstrates two typical coding schemes tested in the experiment. The values to the left of slashes belong to a subject who was sensitive to electrotactile stimulations of lower intensity, while the values to the right of the slashes belong to another subject who could recognise electrotactile stimulations of higher intensity. The preparation and testing of suitable parameters cost about 30-40 minutes on each subject to ensure a comfortable and effortless perception of the 9 stimulation levels.

\section{B. Number of attempts (NoAs)}

The average number of attempts (NoAs) per object in different conditions are shown in Figure. 3(a). When grasping the lightest object, the subjects' learning performance in different 
TABLE I

CODING SCHEME OF ELECTROTACTILE FEEDBACK

\begin{tabular}{llccc}
\hline Stimulation parameter & $\begin{array}{c}\text { Amplitude } \\
(\mathrm{mA})\end{array}$ & $\begin{array}{c}\text { Frequency } \\
\text { (Hz) }\end{array}$ & $\begin{array}{c}\text { Pulse width } \\
\text { (us) }\end{array}$ \\
\hline \multirow{3}{*}{ Channel 1 } & Level 1 & $3 / 3$ & $10 / 10$ & $50 / 180$ \\
& Level 2 & $2 / 2$ & $30 / 61$ & $50 / 50$ \\
& Level 3 & $2 / 2$ & $45 / 62$ & $70 / 50$ \\
\hline \multirow{3}{*}{ Channel 2 2 } & Level 4 & $2 / 3$ & $10 / 10$ & $40 / 180$ \\
& Level 5 & $2 / 2$ & $25 / 60$ & $40 / 50$ \\
& Level 6 & $3 / 2$ & $35 / 61$ & $60 / 45$ \\
\hline \multirow{3}{*}{ Channel 3 3 } & Level 7 & $1 / 3$ & $10 / 10$ & $20 / 160$ \\
& Level 8 & $1 / 2$ & $20 / 56$ & $20 / 60$ \\
& Level 9 & $1 / 2$ & $35 / 58$ & $20 / 100$ \\
\hline
\end{tabular}

feedback conditions was similar. Across the objects, subjects spent comparable NoAs in VF and EF conditions. However, it took approximately twice NoAs to grasp a heavier object (medium, heavy) in NF condition comparing with the NoAs of the other two conditions. The overall average NoAs across conditions as shown in Figure. 3(b) also indicate the same information that the VF and EF help to save about half NoAs comparing with the training in NF condition.

\section{Duration of training (DoT) and Duration of an attempt (DoaA)}

The average duration of training (DoT) as shown in Fig. 4 presents a similar trend with the NoAs in Fig. 3(a). It is reasonable that the DoT is positively correlated with the NoA. The DoT of three feedback conditions were similar when grasping the light object. The VF and EF conditions spent less than $60 \%$ of the training time of the NF condition when grasping the medium object and the heavy object. Additionally, the VF and EF showed a more consistent performance than the NF condition given the smaller standard deviations.

Fig. 5 presents the average duration of an attempt (DoaA) in different feedback conditions during the testing process. The conditions with feedback (VF and $\mathrm{EF}$ ) took a longer duration to accomplish one attempt than the condition of NF. The condition with EF shows the longest DoaA and the largest standard deviation, while the condition with NF shows the least.

\section{Success rate $(S R)$}

The average success rates (SRs) across different objects and feedback conditions are shown in Fig. 6(a). It can be seen that the SRs of both EF and VF outperform those of NF. The $\mathrm{SR}$ in EF condition is comparable with that in VF condition when grasping object 1 (light) and object 2 (medium) and is even observably higher than the SR in the condition of VF when grasping object 3 (heavy). Fig. 6(b) presents the overall average SR of each condition. Grasping with EF shows the highest SR, and the standard deviations in different feedback conditions are comparable.

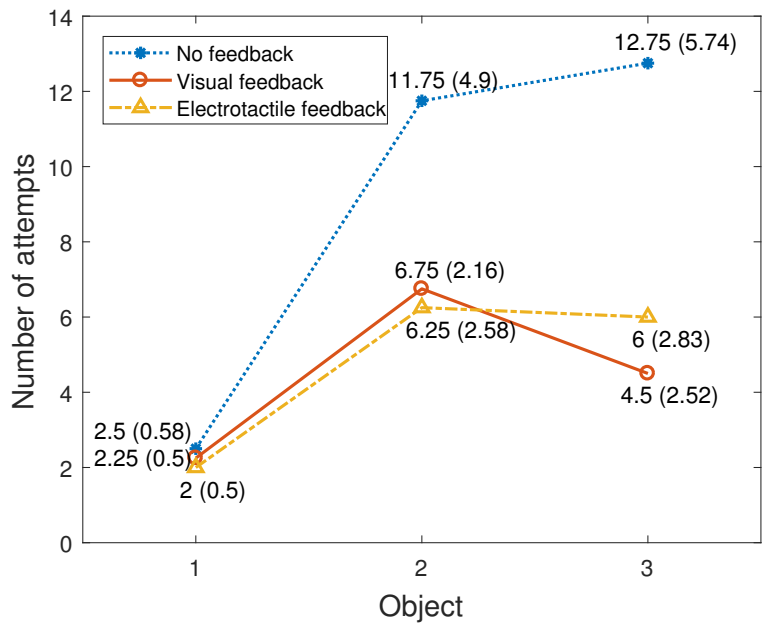

(a) Average number of attempts with the standard deviation of each object in different feedback conditions

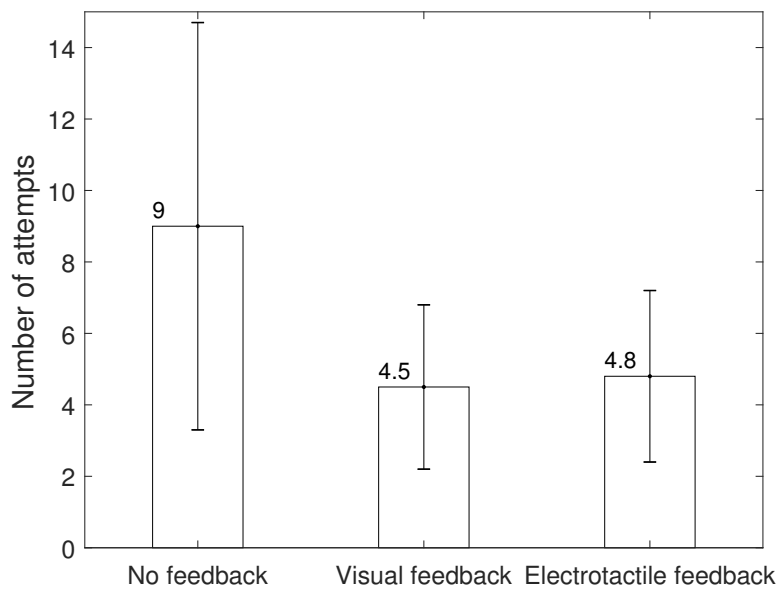

(b) Average number of attempts of all objects in different feedback conditions

Fig. 3. Average number of attempts of training

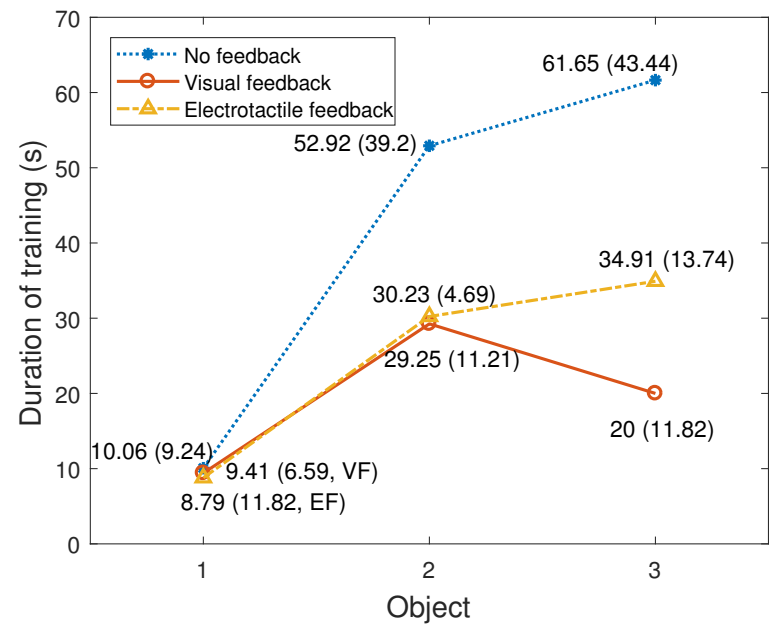

Fig. 4. Average duration of training with the standard deviation of each object in different feedback conditions 


\section{DISCUSSION AND FUTURE WORK}

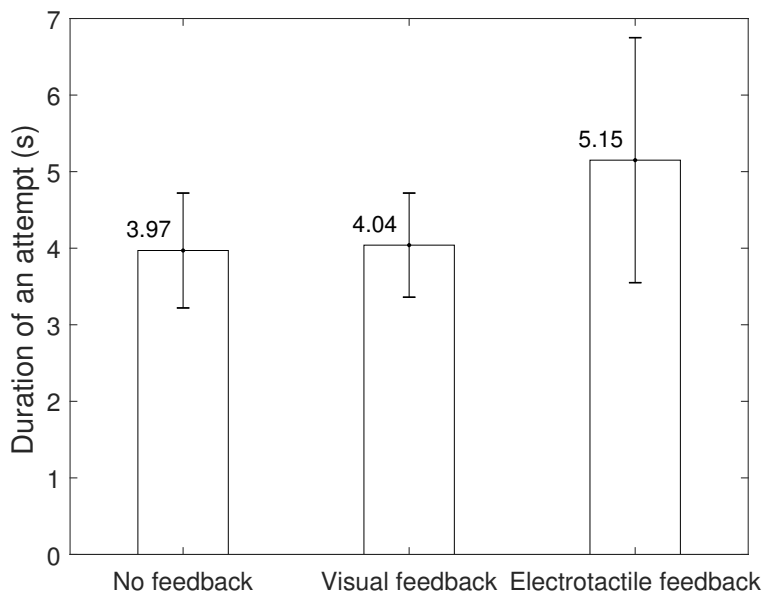

Fig. 5. Average duration of an attempt in different feedback conditions

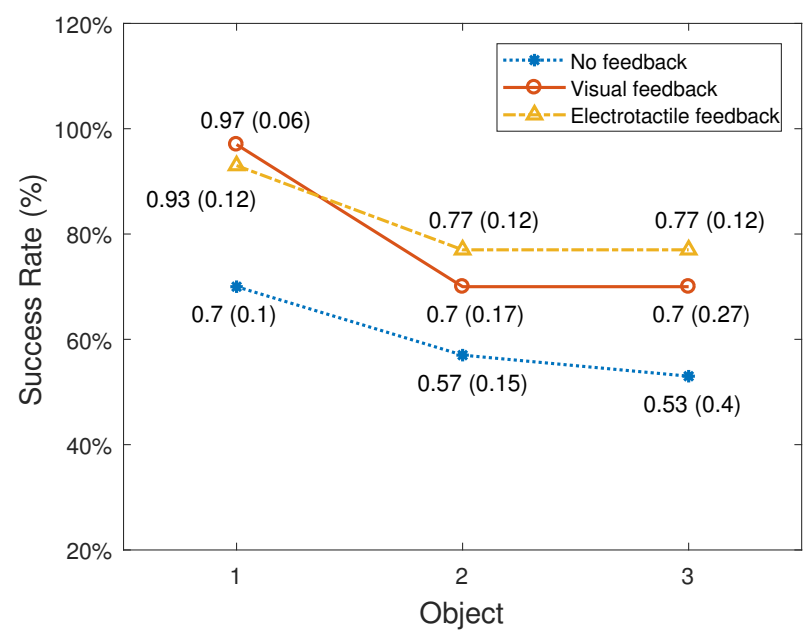

(a) Average success rate with the standard deviation of per object in different feedback conditions

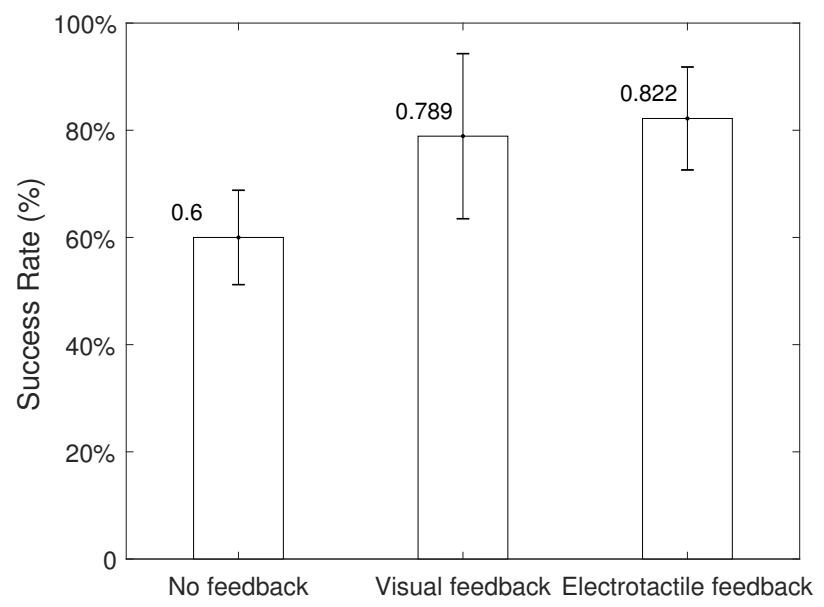

(b) Average success rate in different feedback conditions

Fig. 6. Average success rate during testing process

\section{A. Discussion}

The numerical results acquired from the experiments on ten subjects indicate that both visual feedback and electrotactile feedback contribute to an improved training efficiency and grasp control performance. A plausible explanation is that the incorporation of a feedback module provides a reference for users to adjust their voluntary effort of grasp in comparison with the open-loop control and results in an improved hand grasp in the virtual environment for rehabilitation in terms of the number of attempts, duration of training and success rate. The average number of attempts is the trials required for a success of grasp in the training process, which reflects the learning rate of the users when equipped with different feedback strategies. The absence of a proper feedback module brings about the need for a great number of attempts in training. The heavy training burden without a proper feedback is also revealed by the duration of training when compared to settings with the other two strategies adopted. Both visual and electrotactile feedbacks reduce the required trials by half of the requirements in an open-loop control. Thus the burden of user training is largely reduced, which potentially contributes to a favourable choice by users and the rehabilitation therapists. For example, an average of 2 hours is demanded in our experiments for each subject, most of which is occupied by the training without feedback modules. A replacement of the absent feedback module by either visual feedback or electrotactile feedback is capable of alleviating the timeconsuming training burden.

It can be seen from the experiments that the average time of an attempt with feedback is longer than that of an openloop control, although an improved efficiency is provided by the incorporated feedback. This phenomenon is in accordance with the intuitive understanding that users would conduct straightforward hand grasps without adjustment of their force exertion, which in turn leads to an inferior adaptation to the inevitable variation of muscle contraction during a maintained grasp. As a result, the lack of such a process in conventional grasp control leads to a reduced delay yet with a compromised performance. The increase of the average time of an attempt in a closed-loop control is possible due to the guided selfcorrecting process, which is perceptive and involves the users' voluntary effort in accommodating the dynamic difference between exerted grasp force and desired force. Moreover, despite the negligible delay introduced by the self-correcting process, the usability of the control process is largely improved together with the implicitly improved efficacy, which is partially reflected by a better success rate. A significant improvement of the success rate of grasping 3 objects is seen by an average increase of nearly $15 \%$ and $17 \%$ for the visual feedback and electrotactile feedback, respectively. The improved success rate is in line with supporting and shortening the pathway to grasp control in real life. In summary, both visual and electrotactile feedbacks outperform the open-loop control according to the numerical metrics of grasp evaluation in the whole training and testing phases, while the visual one is more favoured. The force can be controlled in a more stable 
and successful scheme, because of the continuous variation of grasp force according to the numerical hints provided by visual feedback.

Despite that the fact that visual feedback shows superior performance with the least number of attempts and the least duration of training among three candidate feedbacks, it is not practical to utilise the visual hints in clinical applications such as a force bar and a deformation bar to indicate the real-time grasping force. Furthermore, the concentration of users during their prosthesis and virtual hand manipulation is mostly confined to the hand-object interaction without spare capacity left for the visual hint observation. As a result, the application of visual feedback is strongly restricted to a laboratory environment for its non-perceptive nature. Regardless of some degraded metrics in comparison with visual feedback, the electrotactile feedback remains a promising and effective way to provide proper feedback, considering its feasibility in reality and the comparable performance with visual feedback. Let alone the clinical feasibility, an even better success rate is observed on electrotactile feedback in our experiments, which is possibly attributed to a more effective self-correcting process with the prioperception of the hand involved.

In our research, not only the average performance across multiple subjects is concerned, the variation that resides within grasping different objects is also depicted in the experiment results. An intuitive conclusion is that the control of grasping a light object is easier for the users with less training time required yet better success rate. An exception is observed in the electrotactile feedback incorporated heavy object grasp. A better control of grasping the heavy object instead of the medium weighted object is captured as shown in Fig. 6(a). A potential cause of this result could be the perceptive nature of electrotactile stimulation rather than the numerical hint given by visual feedback. The adjustment of hand grasp force between a certain interval with quantitative feedback allows the control without abrupt changes. A large grasp force tends to require more intense muscle contraction, which is not as stable as the light contraction to exert small grasp force. As a result, the prioperception based electrotactile feedback control of grasping a heavy object with a larger force shows an improvement in terms of success rate.

\section{B. Future work}

In addition to the time-consuming process of user training, the testing of suitable stimulation parameters in preparation stage cost quite long time of almost $1 / 3$ of the whole experiment duration, which is due to the huge individual difference of sensitivity to electrotactile stimulation. For example, during the experiment, we found that subjects seem to be much more sensitive to the change of frequency than amplitude and pulse width. A modulation method is expected to simplify the preparation process to save time. Furthermore, the intensity of perceived sensation is not linearly mapped to the stimulation intensity. When the stimulation intensity exceeded a certain level, a subject reported a less intensive perception. To solve this problem, it is necessary to investigate the effect of different stimulation parameters on subjects.
In the current work, only able-bodied subjects were engaged in the grasp control test. Though the high success rate of hand grasps preliminarily verified the feasibility of electrotactile feedback in hand rehabilitation, a discrepancy of physiological conditions may remain between the able-bodied subjects and limb-impaired subjects. Thus, a further evaluation of the electrotactile feedback on targeted subjects with amputation or motor function impairment will be conducted in our future work to validate its clinical usability. Let alone the emphasis on broadening the candidate subjects involved, the proposed virtual platform will be further expanded into a multifunctional system covering a large spectrum of motor function rehabilitation applications by incorporating more constraint properties in hand motion tasks. For example, the sEMG based finger joint angle estimation can be introduced into the rehabilitation platform to train the subject's voluntary control of individual finger movements.

\section{ACKNOWLEDGMENT}

The authors would like to acknowledge all the participants in the experiments, also the support from DREAM project of EU FP7-ICT (Grant No. 611391) and China Scholarship Council (Grant No. 201508060299).

\section{REFERENCES}

[1] J. S. Schofield, K. R. Evans, J. P. Carey, and J. S. Hebert, "Applications of sensory feedback in motorized upper extremity prosthesis: A review," Expert Review of Medical Devices, vol. 11, no. 5, pp. 499-511, 2014.

[2] T. A. Kuiken, G. Li, B. A. Lock, R. D. Lipschutz, L. A. Miller, K. A. Stubblefield, and K. B. Englehart, "Targeted muscle reinnervation for real-time myoelectric control of multifunction artificial arms," Jama, vol. 301, no. 6, pp. 619-628, 2009.

[3] U. Wijk and I. Carlsson, "Forearm amputees' views of prosthesis use and sensory feedback," Journal of Hand Therapy, vol. 28, no. 3, pp. 269-278, 2015.

[4] C. Dietrich, K. Walter-Walsh, S. Preißler, G. O. Hofmann, O. W. Witte, W. H. Miltner, and T. Weiss, "Sensory feedback prosthesis reduces phantom limb pain: proof of a principle," Neuroscience Letters, vol. 507, no. 2, pp. 97-100, 2012.

[5] N. Wake, Y. Sano, R. Oya, M. Sumitani, S.-i. Kumagaya, and Y. Kuniyoshi, "Multimodal virtual reality platform for the rehabilitation of phantom limb pain," in Neural Engineering (NER), 2015 7th International IEEE/EMBS Conference on. IEEE, 2015, pp. 787-790.

[6] Y. Sano, N. Wake, A. Ichinose, M. Osumi, R. Oya, M. Sumitani, S.-i. Kumagaya, and Y. Kuniyoshi, "Tactile feedback for relief of deafferentation pain using virtual reality system: a pilot study," Journal of Neuroengineering and Rehabilitation, vol. 13, no. 1, p. 61, 2016.

[7] M. A. Otaduy, A. Okamura, and S. Subramanian, "Haptic technologies for direct touch in virtual reality," in ACM SIGGRAPH 2016 Courses. ACM, 2016, p. 13.

[8] G. H. Büscher, R. Kõiva, C. Schürmann, R. Haschke, and H. J. Ritter, "Flexible and stretchable fabric-based tactile sensor," Robotics and Autonomous Systems, vol. 63, pp. 244-252, 2015.

[9] H. Lee, K. Park, Y. Kim, and J. Kim, "Durable and repairable soft tactile skin for physical human robot interaction," in Proceedings of the Companion of the 2017 ACM/IEEE International Conference on Human-Robot Interaction. ACM, 2017, pp. 183-184.

[10] X. Wang, T. Xu, S. Dong, S. Li, L. Yu, W. Guo, H. Jin, J. Luo, Z. Wu, and J. M. King, "Development of a flexible and stretchable tactile sensor array with two different structures for robotic hand application," $R S C$ Advances, vol. 7, no. 76, pp. 48 461-48 465, 2017.

[11] M. Mulvey, H. Fawkner, and M. I. Johnson, "An investigation into the perceptual embodiment of an artificial hand using transcutaneous electrical nerve stimulation (tens) in intact-limbed individuals," Technology and Health Care, vol. 22, no. 2, pp. 157-166, 2014.

[12] R. Ackerley and A. Kavounoudias, "The role of tactile afference in shaping motor behaviour and implications for prosthetic innovation," Neuropsychologia, vol. 79, pp. 192-205, 2015. 
[13] D. W. Tan, M. A. Schiefer, M. W. Keith, J. R. Anderson, J. Tyler, and D. J. Tyler, "A neural interface provides long-term stable natural touch perception," Science Translational Medicine, vol. 6, no. 257, p. 257ra138, 2014. [Online]. Available: http: //www.ncbi.nlm.nih.gov/pubmed/25298320

[14] D. J. Tyler, "Restoring the human touch: Prosthetics imbued with haptics give their wearers fine motor control and a sense of connection," IEEE Spectrum, vol. 53, no. 5, pp. 28-33, 2016.

[15] F. Cordella, A. L. Ciancio, R. Sacchetti, A. Davalli, A. G. Cutti, E. Guglielmelli, and L. Zollo, "Literature review on needs of upper limb prosthesis users," Frontiers in Neuroscience, vol. 10, p. 209, 2016.

[16] J. M. Walker, A. A. Blank, P. A. Shewokis, and M. K. OMalley, "Tactile feedback of object slip facilitates virtual object manipulation," IEEE Transactions on Haptics, vol. 8, no. 4, pp. 454-466, 2015.

[17] N. Jorgovanovic, S. Dosen, D. J. Djozic, G. Krajoski, and D. Farina "Virtual grasping: closed-loop force control using electrotactile feedback," Computational and Mathematical Methods in Medicine, vol. 2014, 2014.

[18] M. Aboseria, F. Clemente, L. F. Engels, and C. Cipriani, "Discrete vibro-tactile feedback prevents object slippage in hand prostheses more intuitively than other modalities," IEEE Transactions on Neural Systems and Rehabilitation Engineering, 2018.

[19] I. Saunders and S. Vijayakumar, "The role of feed-forward and feedback processes for closed-loop prosthesis control," Journal of Neuroengineering and Rehabilitation, vol. 8, no. 1, p. 60, 2011.

[20] L. A. Miller, K. A. Stubblefield, R. D. Lipschutz, B. A. Lock, and T. A. Kuiken, "Improved myoelectric prosthesis control using targeted reinnervation surgery: a case series," IEEE Transactions on Neural Systems and Rehabilitation Engineering, vol. 16, no. 1, pp. 46-50, 2008.

[21] J. E. Cheesborough, L. H. Smith, T. A. Kuiken, and G. A. Dumanian, "Targeted muscle reinnervation and advanced prosthetic arms," in Seminars in Plastic Surgery, vol. 29, no. 1. Thieme Medical Publishers, 2015 , p. 62.

[22] K. Takeda, G. Tanino, and H. Miyasaka, "Review of devices used in neuromuscular electrical stimulation for stroke rehabilitation," Medical Devices (Auckland, NZ), vol. 10, p. 207, 2017.

[23] R. J. Onesti, W. Tompkins, J. Webster, and J. Wertsch, "Design of a portable electrotactile stimulator for sensory substitution applications," in Engineering in Medicine and Biology Society, 1989. Images of the Twenty-First Century., Proceedings of the Annual International Conference of the IEEE Engineering in. IEEE, 1989, pp. 1439-1440.

[24] A. Ninu, S. Dosen, S. Muceli, F. Rattay, H. Dietl, and D. Farina, "Closed-loop control of grasping with a myoelectric hand prosthesis: Which are the relevant feedback variables for force control?" IEEE Transactions on Neural Systems and Rehabilitation Engineering, vol. 22, no. 5, pp. 1041-1052, 2014.

[25] M. F. Levin, P. L. Weiss, and E. A. Keshner, "Emergence of virtual reality as a tool for upper limb rehabilitation: incorporation of motor control and motor learning principles," Physical Therapy, vol. 95, no. 3, pp. 415-425, 2015.

[26] S. LeBlanc, K. Paquin, K. Carr, and S. Horton, "Non-immersive virtual reality for fine motor rehabilitation of functional activities in individuals with chronic stroke: a review," Aging Sci, vol. 1, no. 105, p. 2, 2013.

[27] D. Pamungkas and K. Ward, "Electro-tactile feedback system to enhance virtual reality experience," International Journal of Computer Theory and Engineering, vol. 8, no. 6, pp. 465-470, 2016.

[28] S. Dosen, M. Markovic, M. Strbac, V. Kojić, G. Bijelić, T. Keller, D. Farina et al., "Multichannel electrotactile feedback with spatial and mixed coding for closed-loop control of grasping force in hand prostheses," IEEE Transactions on Neural Systems and Rehabilitation Engineering, vol. 25, no. 3, pp. 183-195, 2017.

[29] Y. Fang, H. Liu, G. Li, and X. Zhu, "A multichannel surface emg system for hand motion recognition," International Journal of Humanoid Robotics, vol. 12, no. 02, p. 1550011, 2015.

[30] Y. Fang, D. Zhou, K. Li, and H. Liu, "Interface prostheses with classifierfeedback-based user training," IEEE Transactions on Biomedical engineering, vol. 64, no. 11, pp. 2575-2583, 2017.

[31] K. Li, Y. Fang, Y. Zhou, and H. Liu, "Non-invasive stimulation-based tactile sensation for upper-extremity prosthesis: a review," IEEE Sensors Journal, vol. 17, no. 9, pp. 2625-2635, 2017.

[32] Y. Zhou, Y. Fang, K. Gui, K. Li, D. Zhang, and H. Liu, "semg biasdriven functional electrical stimulation system for upper-limb stroke rehabilitation," IEEE Sensors Journal, vol. 18, no. 16, pp. 6812-6821, 2018.

[33] L. Baker, D. McNeal, L. Benton, B. Bowman, and R. Waters, "Neuromuscular electrical stimulation: A practical guide. downey, ca: Los amigos research and education institute," 1993.
[34] "Hand physics controller," https://assetstore.unity.com/packages/tools/ physics/hand-physics-controller-21105, accessed Aug. 2018.

[35] D. Yang, J. Zhao, Y. Gu, L. Jiang, and H. Liu, "Estimation of hand grasp force based on forearm surface emg," in Mechatronics and Automation, 2009. ICMA 2009. International Conference on. IEEE, 2009, pp. 17951799.

[36] K. Li, Y. Fang, Y. Zhou, J. Zhao, and H. Liu, "Haptics model for human fingertips based on gaussian distribution."

[37] S. Calinon, F. Guenter, and A. Billard, "On learning, representing, and generalizing a task in a humanoid robot," IEEE Transactions on Systems, Man, and Cybernetics, Part B (Cybernetics), vol. 37, no. 2, pp. 286-298, 2007.

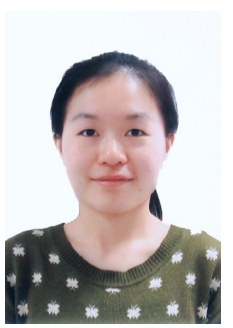

Kairu Li (S'15) received the B.S. degree in Automation and the M.S. degree in Control Engineering and Control Theory from Dalian University of Technology, China, in 2012 and 2015. She is currently pursuing the Ph.D. degree under the supervision of Prof. H. Liu, in the School of Computing from University of Portsmouth, UK. Her research interests include wearable sensor systems, electrotactile stimulation, sensory feedback in prosthetics and rehabilitation.

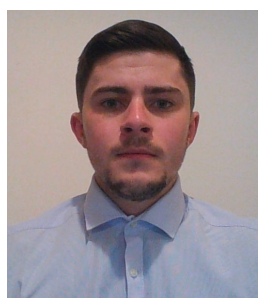

Peter Boyd received a B.Sc. in Computer Science from the University of Portsmouth, UK in 2015. He is currently studying for a Ph.D under the supervision of Prof. H.Liu at the University of Portsmouth, UK. His Research interests include biological signal processing, pattern recognition and virtual systems for rehabilitation.

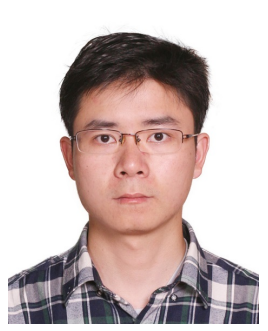

Yu Zhou (S'16) received the B.S. degree from the College of Mechanical and Electrical Engineering, Central South University, Changsha, China, in 2014. $\mathrm{He}$ is currently working toward the Ph.D. degree in the School of Mechanical Engineering, Shanghai Jiao Tong University, Shanghai, China. His research interests include intelligent system, biological signal processing and electrical stimulation.

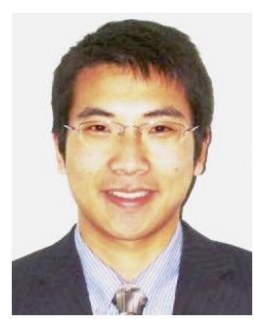

Zhaojie Ju (M'08-SM'16) received the Ph.D. degree in intelligent robotics from the University of Portsmouth, Portsmouth, U.K., in 2010. He is currently a Reader in Machine Learning and Robotics in the School of Computing, University of Portsmouth. He previously held research appointments at the University College London and University of Portsmouth, UK. His research interests include machine intelligence, pattern recognition and their applications on human motion analysis, humanrobot interaction and collaboration and robot skill learning. He has authored or co-authored over 110 publications in journals, book chapters and conference proceedings and received four best paper awards and one Best AE Award in ICRA2018. Dr. Ju is an Associate Editor of the IEEE TRANSACTIONS ON CYBERNETICS and INTERNATIONAL JOURNAL OF HUMANOID ROBOTICS. 


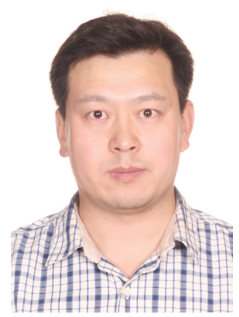

Honghai Liu (M'02-SM'06) received the Ph.D. degree in robotics from Kings College London, London, U.K., in 2003. He joined the University of Portsmouth, Portsmouth, U.K., in September 2005. He previously held the position of research appointments with the Universities of London and Aberdeen, as well as Project Leader appointments in the large-scale industrial control and system integration industry. His current research interests include biomechanics, intelligent sensing, pattern recognition, intelligent video analytics and wearable robotics and their practical applications with an emphasis on approaches that could make contributions to the intelligent connection of perception to action using contextual information. He has published numerous peerreviewed international journal and conference papers and received four best paper awards. Dr. Liu is an Associate Editor of the IEEE TRANSACTIONS ON INDUSTRIAL INFORMATICS and the IEEE TRANSACTIONS ON HUMAN-MACHINE SYSTEMS. 